\title{
Ex vivo mRNA expression of toll-like receptors during latent tuberculosis infection Running head: mRNA expression of toll-like receptors
}

\author{
Birhan Alemnew ${ }^{1}$, Soren Hoff ${ }^{2}$, Tamrat Abebe ${ }^{3}$, Markos Abebe $^{4}$, Abraham Tekolla ${ }^{4}$, \\ Rawleigh Howe ${ }^{4}$, and Liya Wassie ${ }^{4}$ \\ ${ }^{1}$ Woldia University \\ ${ }^{2}$ Statens Serum Institut \\ ${ }^{3}$ Addis Ababa University \\ ${ }^{4}$ Armauer Hansen Research Institute
}

October 20, 2020

\begin{abstract}
Background Understanding immune mechanisms, particularly the role of innate immune markers during latent TB infection remains elusive. The main objective of this study was to evaluate mRNA gene expression patterns of toll-like receptors (TLRs) as correlates of immunity during latent TB infection and further infer their roles as potential diagnostic biomarkers. Methods Messenger RNA (mRNA) were analysed in a total of 64 samples collected from apparently healthy children and adolescents, who were latently infected with tuberculosis $(n=32)$ or non-infected $(n=32)$ and were selected using purposive sampling to compare the relative expressions of selected TLRs (TLR-1, TLR-2, TLR-4, TLR-6 and TLR-9), using quantitative real-time polymerase chain reaction (qRT-PCR). Specific primers and florescent labelled probes were used to detect the expression of these markers in peripheral blood; a comparative threshold cycle method was used to describe fold change in the relative expression of TLR genes. Data were analysed using Graph-Pad Prism 7.01 for Windows and a p-value less than 0.05 was considered statistically significant. Results An increased mean fold change in the relative expression of TLR-2 and TLR-6 mRNA was observed in LTBI groups relative to non-LTBI groups $(\mathrm{p}<0.05)$, whereas a slight fold decrease was observed for TLR-1 gene. Conclusions An increased mRNA expression of TLR-2 and TLR-6 was observed in latently infected individuals relative to those non-infected, possibly indicating the roles these biomarkers play in sustenance of the steady state interaction between the dormant TB bacilli and host immunity.
\end{abstract}

\section{Hosted file}

Birhan Alemnew et al._Full MS.pdf available at https://authorea.com/users/368665/articles/ 487739-ex-vivo-mrna-expression-of-toll-like-receptors-during-latent-tuberculosisinfection-running-head-mrna-expression-of-toll-like-receptors 